\title{
Hit Time and Hit Position Reconstruction in the J-PET Detector Based on a Library of Averaged Model Signals
}

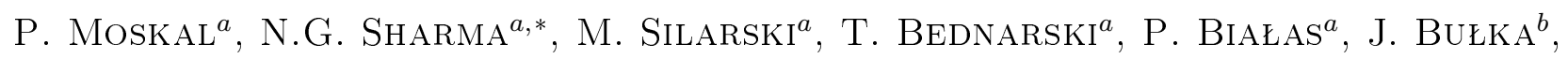

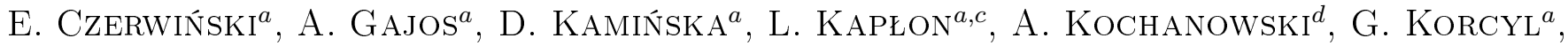

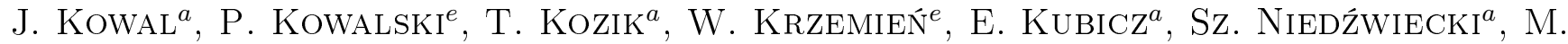

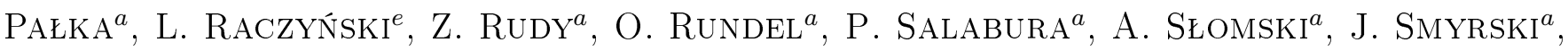

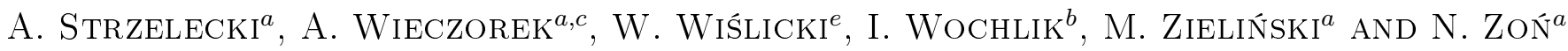

${ }^{a}$ Faculty of Physics, Astronomy and Applied Computer Science, Jagiellonian University, 30-348 Kraków, Poland

${ }^{b}$ Department of Automatics and Bioengineering AGH, Krakow, Poland

${ }^{c}$ Institute of Metallurgy and Materials Science of Polish Academy of Sciences, 30-059 Kraków, Poland

${ }^{d}$ Faculty of Chemistry, Jagiellonian University, 30-060 Kraków, Poland

${ }^{e}$ Świerk Computing Centre, National Centre for Nuclear Research, 05-400 Otwock-Świerk, Poland

In this article we present a novel method of hit time and hit position reconstruction in long scintillator detectors. We take advantage of the fact that for this kind of detectors amplitude and shape of registered signals depend strongly on the position where particle hits the detector. The reconstruction is based on determination of the degree of similarity between measured and averaged signals stored in a library for a set of well-defined positions along the scintillator. Preliminary results of validation of the introduced method with experimental data obtained by means of the double strip prototype of the J-PET detector are presented.

DOI: 10.12693/APhysPolA.127.1495

PACS: 87.57.uk, 07.05.Kf

\section{Introduction}

Recently a new concept of large acceptance Jagiellonian PET (J-PET) system was proposed [1-4]. Unlike all the commercial PET devices using inorganic scintillators as radiation detectors [5-8] (usually these are the BGO, LSO or LYSO crystals) J-PET is based on polymer scintillators. This technique offers improvement of the time of flight (TOF) resolution ${ }^{\dagger}$ and also constitutes a promising solution for a whole-body PET imaging. In the case of J-PET annihilation gamma quanta are registered by means of long scintillator strips read out from both ends by photomultipliers. This allows for the determination of position and time of the gamma quanta reaction based predominantly on the time measurement. Therefore, to fully exploit the potential of this solution, it requires the elaboration of a new hit position and TOF reconstruction methods [10]. Recently, one method of reconstruction in scintillator detectors based on the comparison of registered signals with respect to a library of synchronized model signals collected for a set of welldefined positions along the scintillator [11] is published.

In this article we present similar method, however the comparison of measured signal is done with averaged model signals determined as a function of position along the scintillator. This approach speeds up significantly

\footnotetext{
*corresponding author; e-mail: tanu8513@gmail.com

†Presently the best TOF resolution was achieved with LSO crystals and amounts to about $400 \mathrm{ps}$ [9].
}

the reconstruction in comparison to the previously used method.

\section{Library of synchronized model signals}

In order to create the library of model signals, we have used the setup composed of two BC-420 [12] scintilators with dimensions $300 \times 19 \times 5 \mathrm{~mm}^{3}$ and Hamamatsu photomultipliers R4998 [13] connected optically to their most distant ends via optical gel EJ-550 [14]. General scheme of experimental setup used to build the library of signals is shown in Fig. 1.

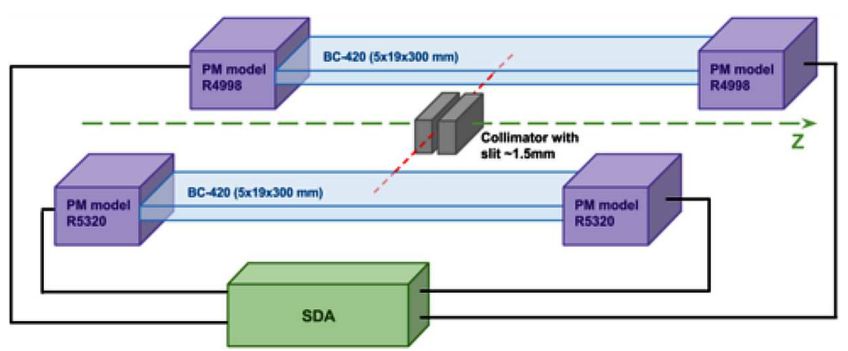

Fig. 1. Scheme of experimental setup where abbrevations PM and SDA denote photomultiplier and serial data analyser (SDA6000A), respectively.

The two detectors were irradiated with a collimated beam of annihilation quanta $(\mathrm{FWHM} \approx 1.5 \mathrm{~mm})$ in steps of $3 \mathrm{~mm}$. The ${ }^{22} \mathrm{Na}$ source was placed inside a lead collimator which could be moved along the scintillator by using a dedicated mechanical system. For each position a high statistics of sampled signals with interval of $100 \mathrm{ps}$ was collected by means of SDA. A coincident registration 
of signals from both detectors allowed for the photomultipliers noise suppression and the selection of annihilation gamma quanta.

Exemplary sampled signal is shown in Fig. 2a. To create a background-free library, first we have corrected all the signals for pedestal. For every signal the average value of voltage was calculated in the noise region shown in Fig. 2a by encircled red area. This computed average value was then used for pedestal correction for that particular signal. An exemplary signal after correction is presented in Fig. $2 b$.

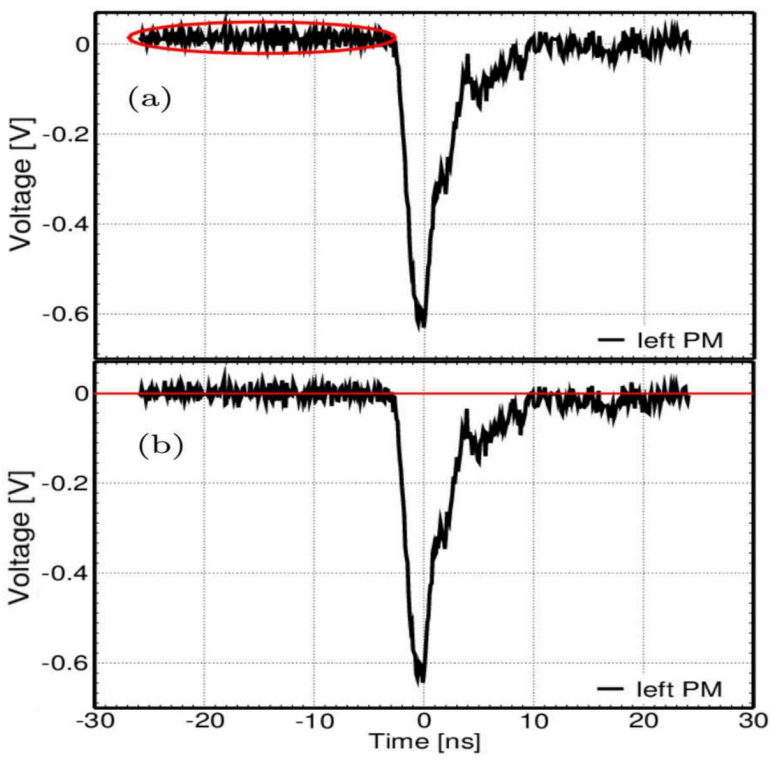

Fig. 2. (a) Measured signal from the database. The same signal after pedestal correction.

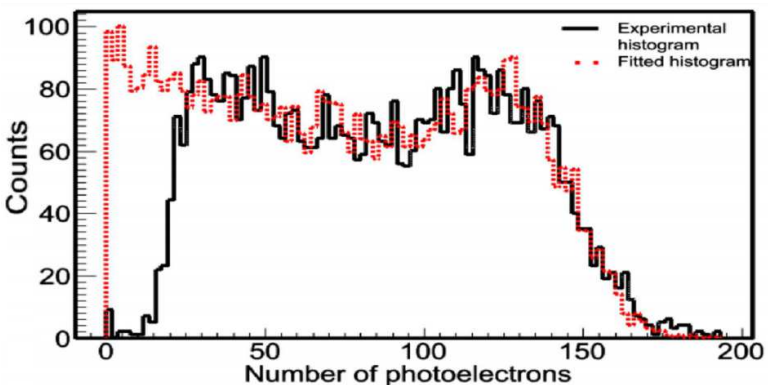

Fig. 3. Distribution of number of photoelectrons obtained from signals measured irradiating the scintillator strip at central position (solid black histogram). Dashed red histogram represents theoretical distribution [15] fitted to the experimental data using normalization and energy calibration constants as free parameters. Details of the fitting procedure are described in Ref. [16].

After pedestal correction, signals building the library were selected based on number of registered photoelectrons such that it is constructed from events with the high energy deposition.

In Fig. 3 we show an example of such spectrum obtained at central position. For every position we have cut all the signals for which the number of registered photoelectrons is lower than half of the number of photoelectrons corresponding to the Compton edge for $511 \mathrm{keV}$ gamma quanta.

Finally, database signals were synchronized by shifting their time scales in such a way that time of the gamma quantum hit inside the detector is the same for all events in the library.

The shape of a model signal for each position from the database is determined by averaging pedestal corrected and synchronized signals. More details on model signals determination are given in the next section of this article.

\section{Determination of the shape of model signals}

Function describing the approximate shape of the model signal was determined by averaging measured signals for a given hit position. Calculated average for signals registered simultaneously at both ends of the scintillator was treated as a reference in order to align all the database signals measured for a particular position, as it is shown in Fig. 4a and b.

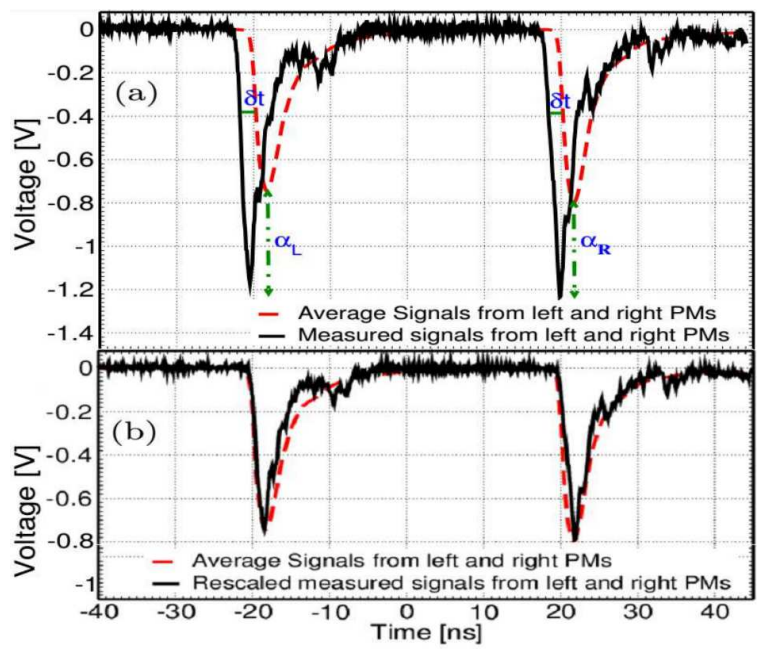

Fig. 4. (a) Example of a database signal before alignment to the average. Black curve represents the measured signal, while the average signal is shown by red dashed curve. (b) The same signal after rescaling.

Such alignment is necessary to account for the spread of signals in amplitude and time. To perform signal's alignment in the database, we have defined for each measured signal at a given position a $\chi^{2}$ value. It was computed by comparing leading edge of the database and average signals. Comparison was performed by taking into account signal registered at the left and right side of scintillator simultaneously. The $\chi^{2}$ was calculated as a function of three parameters $\delta t, \alpha_{\mathrm{L}}$ and $\alpha_{\mathrm{R}}$ :

$$
\begin{gathered}
\chi^{2}\left(\delta t, \alpha_{\mathrm{L}}, \alpha_{\mathrm{R}}\right)=\sum_{i=1}^{n} \frac{t_{\mathrm{AvgL}}\left(V_{i}\right)-t_{\mathrm{dbL}}\left(\left(\alpha_{\mathrm{L}} V_{i}\right)-\delta t\right)^{2}}{n} \\
+\sum_{i=1}^{m} \frac{\left(t_{\mathrm{AvgR}}\left(V_{i}\right)-t_{\mathrm{dbR}}\left(\left(\alpha_{\mathrm{R}} V_{i}\right)-\delta t\right)\right)^{2}}{m}
\end{gathered}
$$

$\delta t$ is a time shift for sample points along time axis 
and $\alpha_{\mathrm{L}}, \alpha_{\mathrm{R}}$ are normalisation factors for both signals (left and right) registered at both ends of scintillator. $t_{\mathrm{AvgL}}\left(V_{i}\right)$ and $t_{\mathrm{AvgR}}\left(V_{i}\right)$ denote time for model signal computed at left and right side for voltage $V_{i}$ at their leading edge. $t_{\mathrm{dbL}}\left(\alpha_{\mathrm{L}} V_{i}\right)$ and $t_{\mathrm{dbR}}\left(\alpha_{\mathrm{R}} V_{i}\right)$ is the time computed for rescaled left and right signals at their leading edge, respectively. By minimization of the $\chi^{2}$ value we obtained the best alignment between two compared signals and each database signal was rescaled using fit parameters $\alpha_{\mathrm{L}}, \alpha_{\mathrm{R}}$ and $\delta t$ giving the lowest $\chi^{2}$. Next, average of these rescaled signals was computed again leading to the model signal determination and the whole procedure was repeated until the changes were negligible. Example of model signals obtained at three different positions is shown in Fig. 5.

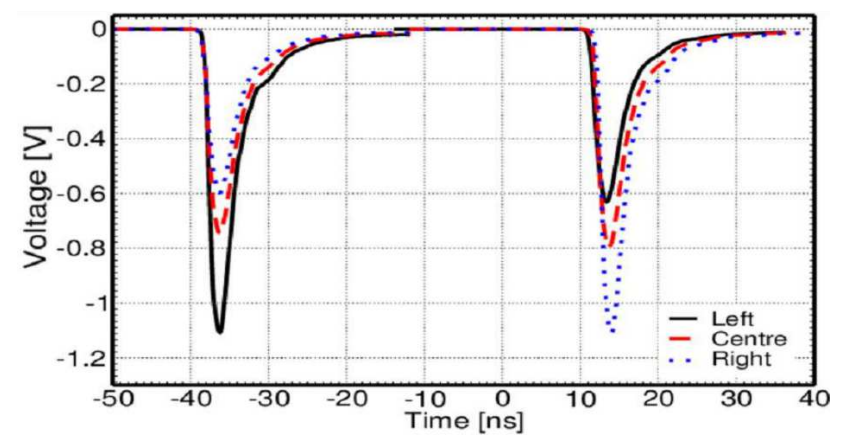

Fig. 5. Sample of model signals at three different positions: solid black line represents the left side of the scintillator, dashed red line center and dotted blue line the right side of scintillator.

\section{Validation and optimization of the time and position reconstruction}

In order to reconstruct hit position of gamma quanta in the detector the measured signals are compared with each model signal from the library. This is done by minimizing $\chi^{2}$ value calculated at the leading edges of measured and model signals. Validation and optimization of this reconstruction method has been performed by utilizing the signals gathered at known positions with the same experimental setup which was used to build the library. In order to simulate the response of front-end electronics we have determined the times corresponding to predefined constant level thresholds [17]: $-60,-120$, 180 , and $-240 \mathrm{mV}$. Analogously, we have chosen also four constant fraction thresholds to sample signals at: $1 / 8 A_{i}$, $2 / 8 A_{i}, 3 / 8 A_{i}, 4 / 8 A_{i}$, where $A_{i}$ is the amplitude of signal which is smallest among all four compared signals. To optimize the reconstruction we have considered two posibilities: 1 . when $\chi^{2}$ is a function of the time shift $\delta t$ only

$$
\begin{gathered}
\chi^{2}(\delta t)=\sum_{i=1}^{4}\left[t_{\mathrm{MdL}}\left(V_{i}\right)-t_{\mathrm{dbL}}\left(V_{i}-\delta t\right)\right]^{2} \\
+\sum_{i=1}^{4}\left[t_{\mathrm{MdR}}\left(V_{i}\right)-t_{\mathrm{dbR}}\left(V_{i}-\delta t\right)\right]^{2},
\end{gathered}
$$

2. when $\chi^{2}$ depends on $\delta t$ and normalisation factors $\alpha_{\mathrm{L}}$ and $\alpha_{\mathrm{R}}$

$$
\begin{aligned}
& \chi^{2}\left(\delta t, \alpha_{\mathrm{L}}, \alpha_{\mathrm{R}}\right)=\sum_{i=1}^{4}\left(t_{\mathrm{MdL}}\left(V_{i}\right)-t_{\mathrm{dbL}}\left(\left(\alpha_{\mathrm{L}} V_{i}\right)-\delta t\right)\right)^{2} \\
& +\sum_{i=1}^{4}\left(t_{\mathrm{MdR}}\left(V_{i}\right)-t_{\mathrm{dbR}}\left(\left(\alpha_{\mathrm{R}} V_{i}\right)-\delta t\right)\right)^{2} .
\end{aligned}
$$

$t_{\mathrm{MdL}}\left(V_{i}\right), t_{\mathrm{MdR}}\left(V_{i}\right)$ and $t_{\mathrm{dbL}}\left(V_{i}\right), t_{\mathrm{dbR}}\left(V_{i}\right)$ denote times determined at left and right side of the scintillator at threshold $V_{i}$ for model and registered signals, respectively. The reconstructed hit position is the position of most similar signal from the library with respect to measured signal (i.e. model signal for which $\chi^{2}$ is minimal ${ }^{\ddagger}$ ).

The time of particle interaction is determined as a relative time between the measured signal and the most similar one from the library. This provides also determination of the gamma quantum time of flight (TOF) [11]:

$t_{\mathrm{fStr}}=\delta t_{\mathrm{fStr}}, \quad t_{\mathrm{sStr}}=\delta t_{\mathrm{sStr}}, \quad \mathrm{TOF}=t_{\mathrm{sStr}}-t_{\mathrm{fStr}}$, where $\delta t$ denotes shift in time for which the computed $\chi^{2}$ defined in Eqs. (2) and (3) is lowest.

An example of the $\chi^{2}$ distribution calculated according to Eq. (2) for one of signals sampled with constant levels and measured at central position of the strip is shown in Fig. 6. One can see a clear minimum corresponding to $z \approx 150 \mathrm{~mm}$.

Present version of reconstruction procedure do not take into account the measurement uncertainties. Therefore, the $\chi^{2}$ values are treated as arbitrary and errors of fitted parameters are determined directly from distributions of differences between reconstructed and true values of time or position. Figure $7 \mathrm{a}$ and $\mathrm{b}$ shows distributions of differ-

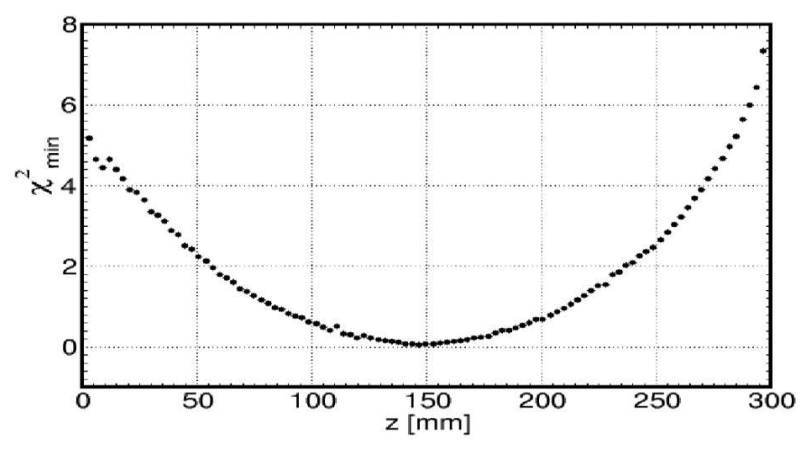

Fig. 6. Example of the $\chi^{2}$ distribution defined in Eq. 2.

ences between the true and reconstructed position using $\chi^{2}$ defined by Eq. 2 for constant fraction and constant levels discrimination, respectively. Resolution of position reconstruction is determined by fitting a Gauss function to the presented distribution and obtained results give: $\sigma_{z}=9.7 \pm 0.3 \mathrm{~mm}$ for constant fraction sampling and $\sigma_{z}=9.6 \pm 0.3 \mathrm{~mm}$ for constant levels.

In case when $\chi^{2}$ is a function of $\delta t, \alpha_{\mathrm{L}}$ and $\alpha_{\mathrm{R}}$ (Eq. (2)) obtained position resolution amounts to $\sigma_{z}=$

$\ddagger$ Since the degree of similarity is represented by the $\chi^{2}$ value. 


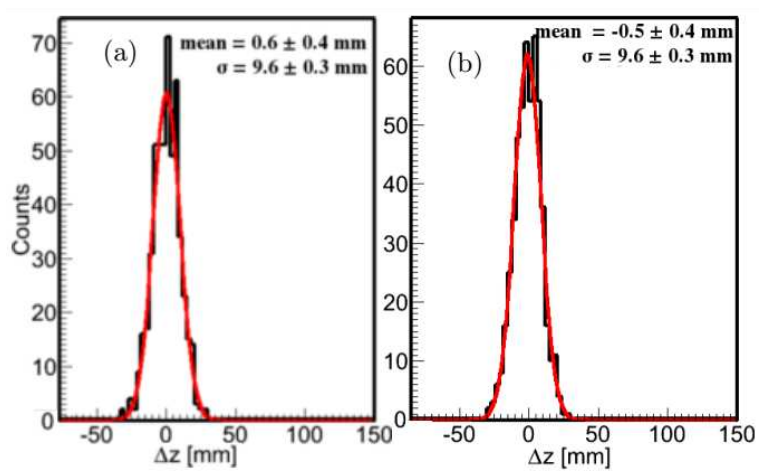

Fig. 7. Distribution of differences between the true and reconstructed position $\Delta z$ for signals measured at $z=150 \mathrm{~mm}$ for (a) constant fractions (b) constant levels.

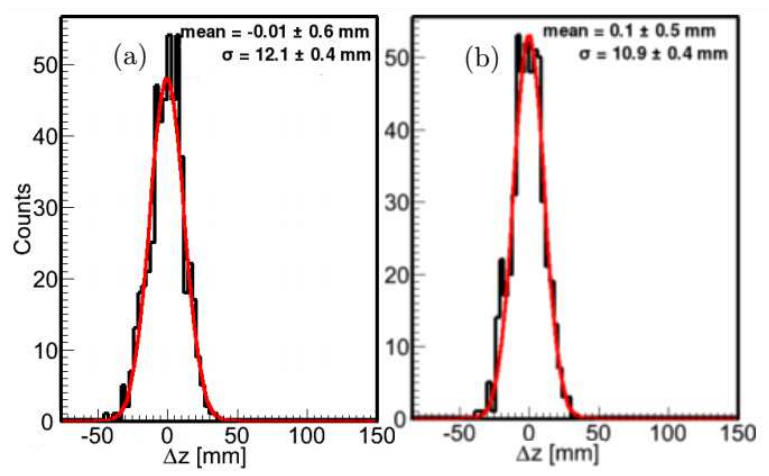

Fig. 8. Distribution of differences between the true and reconstructed position $\Delta z$ for signals measured at $z=150 \mathrm{~mm}$ for (a) constant fractions (b) constant levels.

$12.1 \pm 0.4 \mathrm{~mm}$ and $10.9 \pm 0.4 \mathrm{~mm}$ for constant fraction and constant level method, respectively. Corresponding $\Delta z$ distributions are shown in Fig. 8. These resolutions were determined for signals measured at several positions along the scintillator as it is shown in Fig. 9. The results indicate that this resolution does not change much with position. Results for TOF reconstruction for signals measured at $\mathrm{z}=150 \mathrm{~mm}$ are shown in Fig. 10. Corresponding

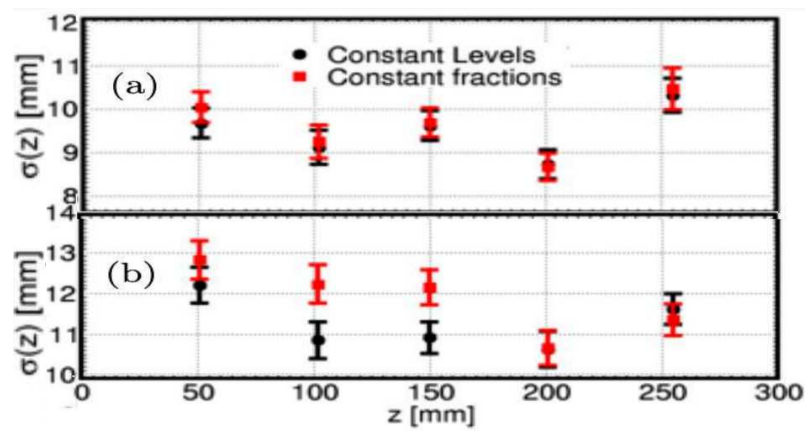

Fig. 9. Position resolution as a function of the position of gamma quantum interaction for the case when (a) $\chi^{2}$ is a function of $\delta t$ only (b) $\chi^{2}$ is a function of $\delta t$, $\alpha_{\mathrm{L}}$ and $\alpha_{\mathrm{R}}$.
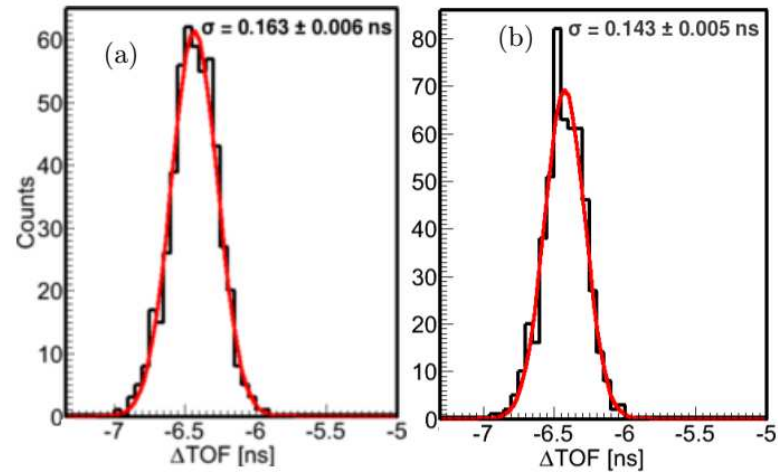

Fig. 10. Distribution of differences between the true and reconstructed TOF for events registered at $z=$ $150 \mathrm{~mm}$. The reconstruction was done using $\chi^{2}$ as a function of $\delta t$ with signal sampling at (a) constant fraction and (b) at constant levels.
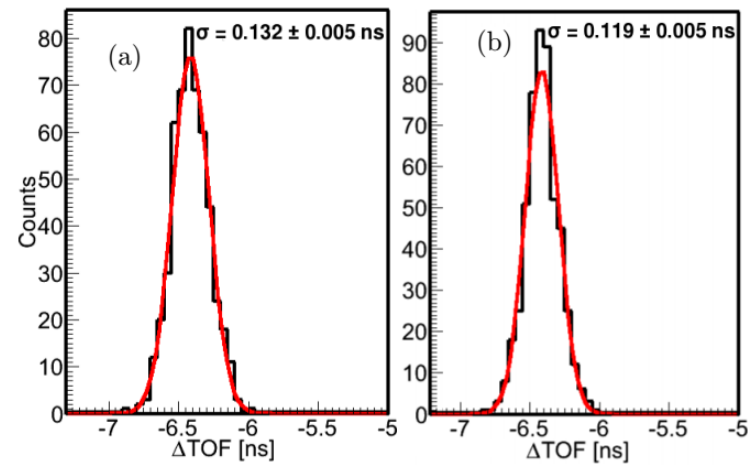

Fig. 11. Distribution of differences between the true and reconstructed TOF for signals measured at $z=$ $150 \mathrm{~mm}$. The reconstruction was done using $\chi^{2}$ as a function of $\delta \mathrm{t}, \alpha_{L}$, and $\alpha_{R}$, and with signal sampling at (a) constant fraction (b) constant levels.

resolutions are in this case equal to $\sigma_{\mathrm{TOF}} \approx 163 \mathrm{ps}$ for constant fractions sampling and $\sigma_{\mathrm{TOF}} \approx 143 \mathrm{ps}$ in case of constant levels. As it is shown in Fig. 11 results obtained for $\chi^{2}\left(\delta t, \alpha_{\mathrm{L}}, \alpha_{\mathrm{R}}\right)$ amount to $\sigma_{\mathrm{TOF}} \approx 132 \mathrm{ps}$ (constant fractions) and $\sigma_{\mathrm{TOF}} \approx 119 \mathrm{ps}$ (constant levels).

In principle true value of TOF should be equal to zero when source was positioned in the middle between de-

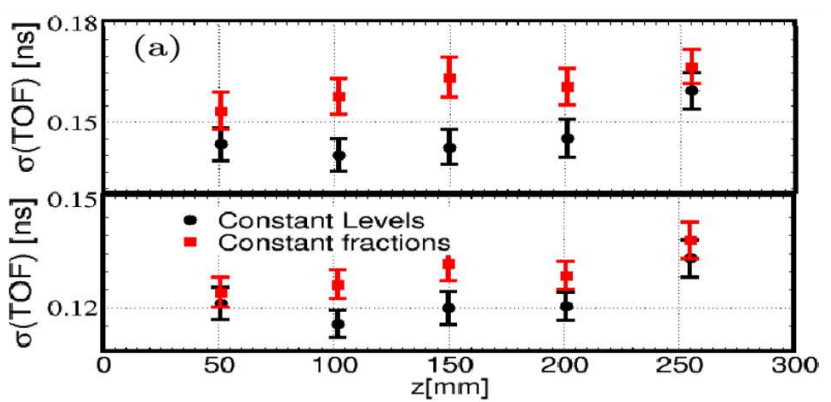

Fig. 12. TOF resolution as a function of position along the scintillator: (a) $\chi^{2}=\chi^{2}(\delta t)$ and (b) $\chi^{2}=$ $\chi^{2}\left(\delta t, \alpha_{\mathrm{L}}, \alpha_{\mathrm{R}}\right)$. 
tection modules. However, due to different time offsets produced by electronics the reconstructed mean values of TOF may be different from zero.

Again, we have performed studies of the TOF resolution as a function of position for both $\chi^{2}$ functions as it is shown in Fig. 12. One can see that the determined resolutions is constant within the error bars over the full length of $30 \mathrm{~cm}$ long scintillator strip.

\section{Summary}

The preliminary results obtained during validation of the reconstruction method introduced in this article show that it is possible to obtain a spatial resolution of about $1.2 \mathrm{~cm}(\sigma)$ for the gamma quanta hit position, and TOF resolution of about $125 \mathrm{ps}(\sigma)$. As regards the position resolution along the detector obtained result is few time worse than achieved by the commercial TOF-PET scanners, However as regards the TOF determination obtained result is better by about a factor of two with respect to resolutions achieved by the commercial TOFPET tomographs characterized by typical field of views of about $16 \mathrm{~cm}$ and TOF resolution of about $230 \mathrm{ps}(\sigma)$ [18]. A further improvement is expected in the future by including measurement uncertainties and possible correlations between the times measured at different thresholds.

\section{References}

[1] P. Moskal, Patents granted in 2014, Nos. EP2454612B1, WO2011008119, EP2454611, WO2011008118.

[2] P. Moskal, P. Salabura, M. Silarski, J. Smyrski, J. Zdebik, M. Zieliński, Bio-Algorithms Med-Syst. 7, 73 (2011).

[3] P. Moskal, T. Bednarski, P. Białas, M. Ciszewska, E. Czerwiński, A. Heczko, M. Kajetanowicz, Ł. Kapłon, A. Kochanowski, G. Konopka-Cupiał, G. Korcyl, W. Krzemień, K. Łojek, J. Majewski, W. Migdal, M. Molenda, Sz. Niedźwiecki, M. Pałka, Z. Rudy, P. Salabura, M. Silarski, A. Słomski, J. Smyrski, J. Zdebik, M. Zieliński, Nucl. Med. Rev. 15, C68 (2012).

[4] P. Moskal, T. Bednarski, P. Białas, M. Ciszewska, E. Czerwiński, A. Heczko, M. Kajetanowicz, Ł. Kapłon, A. Kochanowski, G. KonopkaCupiałG. Korcyl, W. Krzemień, K. Łojek, J. Majewski, W. Migdał, M. Molenda, Sz. Niedźwiecki, M. Pałka, Z. Rudy, P. Salabura, M. Silarski, A. Słomski, J. Smyrski, J. Zdebik, M. Zieliński, Nucl. Med. Rev. 15, C81 (2012).

[5] M. Conti, Phys. Med. 25, 1 (2009).

[6] J.L. Humm, A. Rosenfeld, A. Del Guerra, Eur. J. Nucl. Med. Mol. Imaging 30, 1574 (2003).

[7] J.S. Karp, S. Surti, M.E. Daube-Witherspoon, G. Muehllehner, J. Nucl. Med. 49, 462 (2008).

[8] J.W. Townsend, Ann. Acad. Med. Singapore 22, 133 (2004).
[9] W.W. Moses, S.E. Derenzo, IEEE Trans. Nucl. Sci. 46, 474 (1999).

[10] L. Raczyński, P. Kowalski, W. Wiślicki, T. Bednarski, P. Białas, E. Czerwiński, Ł. Kapłon, A. Kochanowski, G. Korcyl, J. Kowal, T. Kozik, W. Krzemień, E. Kubicz, M. Molenda, I. Moskal, Sz. Niedźwiecki, M. Pałka, M. Pawlik-Niedźwiecka, Z. Rudy, P. Salabura, N.G. Sharma, M. Silarski, A. Słomski, J. Smyrski, A. Strzelecki, A. Wieczorek, M. Zieliński, N. Zoń, Nucl. Instrum. Methods Phys. Res. A 764, 186 (2014); L. Raczyński, P. Kowalski, W. Wiślicki, T. Bednarski, P. Białas, E. Czerwiński, Ł. Kapłon, A. Kochanowski, G. Korcyl, J. Kowal, T. Kozik, W. Krzemień, E. Kubicz, M. Molenda, I. Moskal, Sz. Niedźwiecki, M. Pałka, Z. Rudy, P. Salabura, N.G. Sharma, M. Silarski, A. Słomski, J. Smyrski, A. Strzelecki, A. Wieczorek, M. Zieliński, N. Zoń, Nucl. Instrum. Methods Phys. Res. A 786, 105 (2015).

[11] P. Moskal, N. Zoń, T. Bednarski, P. Białas, E. Czerwiński, A. Gajos, D. Kamińska, Ł. Kapłon, A. Kochanowski, G. Korcyl, J. Kowal, P. Kowalski, T. Kozik, W. Krzemień, E. Kubicz, Sz. Niedźwiecki, M. Pałka, L. Raczyński, Z. Rudy, O. Rundel, P. Salabura, N.G. Sharma, M. Silarski, A. Słomski, J. Smyrski, A. Strzelecki, A. Wieczorek, W. Wiślick, M. Zieliński, Nucl. Instrum. Methods Phys. Res. A 775, 54 (2015).

[12] Saint Gobain Crystals, http://www.crystals.saintgobain.com.

[13] Hamamatsu, http://www.hamamatsu.com.

[14] Scionix, http://www.scionix.nl.

[15] O. Klein, Y. Nishima, Z. Phys. 52, 853 (1929).

[16] P. Moskal, Sz. Niedźwiecki, T. Bednarski, E. Czerwiński, Ł. Kapłon, E. Kubicz, I. Moskal, M. PawlikNiedźwiecka, N.G. Sharma, M. Silarski M. Zieliński, N. Zoń, P. Białas, A. Gajos, A. Kochanowski, G. Korcyl, J. Kowal, P. Kowalski, T. Kozik, W. Krzemień, M. Molenda, M. Pałka, L. Raczyński, Z. Rudy, P. Salabura, A. Słomski, J. Smyrski, A. Strzelecki, A. Wieczorek, W. Wiślicki, Nucl. Instrum. Methods Phys. Res. A 764, 317 (2014).

[17] M. Pałka, T. Bednarski, P. Białas, E. Czerwiński, Ł. Kapłon, A. Kochanowski, G. Korcyl, J. Kowal, P. Kowalski, T. Kozik W. Krzemień, M. Molenda, P. Moskal, Sz. Niedźwiecki, M. Pawlik, L. Raczyński, Z. Rudy, P. Salabura, N.G. Sharma, M. Silarski, A. Słomski, J. Smyrski, A. Strzelecki, W. Wiślicki, M. Zieliński, N. Zoń, Bio-Algorithms Med-Syst. 10, 41 (2014).

[18] V. Bettinardi, M. Danna, A. Savi, M. Lecchi, I. Castiglioni, M. C. Gilardi, H. Bammer, G. Lucignani, F. Fazio, Med. Phys. 38, 5394 (2011). 